\title{
IDENTIFICATION OF THE PROCESS OF DYNAMIC STRETCHING OF THREADS IN WARP KNITTING TECHNOLOGY \\ PART II: EXPERIMENTAL IDENTIFICATION OF THE PROCESS OF STRETCHING THREADS, WITH VERIFICATION OF RHEOLOGICAL MODELS
}

\author{
Aleksandra Prążyńska*, Zbigniew Mikołajczyk \\ Department of Knitting Technology, Lodz University of Technology \\ 116 Żeromskiego St., 90-924 Łódź, Poland \\ E-mail: aleksandra.prazynska@p.lodz.pl, zbigniew.mikolajczyk@p.lodz.pl \\ *corresponding author
}

\begin{abstract}
:
The study is a continuation of the first part of the publication, concerning the theoretical analysis of sensitivity of rheological models of dynamically stretched thread. This part presents the experimental research on the characteristics of stretching forces as a function of time, in the context of comparing the obtained results with theoretical data.

The selected research material was three types of polyester silk threads with linear densities 84, 110 and 334 dtex. During the tests, threads of 400 to $1300 \mathrm{~mm}$ were stretched at speeds in the range of $V \in[0.65,1.47]$ $\mathrm{m} / \mathrm{s}$, which corresponds to the knitting speed $n \in[700,1600]$ courses/min. An original measuring device for empirical identification of dynamic processes of threads stretching was used during the tests. In total, 36 tests variants were performed. The procedures of mathematical equivalence were also worked out for the rheological models of Kelvin-Voigt, Zener and the three-parameter Standard model 2, which enabled the description of threads stretching phenomena in technological processes with the help of generalized rheological model, taking into account the visco-elastic qualities of threads.

Experimental results showed that for the assumed deformation speeds $\varepsilon(t) \in[0.53,4.00] 1 / \mathrm{s}$, the open threeparameter models - Standard model 2 and Zener model most reliably describe the stretching process.
\end{abstract}

\section{Keywords:}

rheological models, dynamic stretching of threads, knitting on warp-knitting machines, empirical verification of rheological model

\section{Introduction}

The publication is a continuation of the issues discussed in the first part of the article ${ }^{[1]}$ concerning the sensitivity of rheological models to input data, in the context of dynamic stretching of threads during the process of knitting on warp knitting machines. In the article, the obtained calculated values are verified and compared with the empirically measured ones, for the four selected multi-parameter rheological models. Empirical tests of stretching processes with respect to knitting on warp knitting machines proved that the open three-parameter models - Standard model 2 and Zener model - most reliably describe the physical phenomena taking place inside the textile visco-elastic bodies.

The novelty of the presented results of experimental verification of multi-parameter rheological models lies in the mathematical and physical interpretation of the phenomena of stretching threads subjected to loadings during knitting processes on warp-knitting machines. A simplified record of the rheological equation of state was formulated for the Kelvin-Voigt model, which is equivalent to the three-parameter Zener model and Standard model 2 in terms of the accepted parameters of viscosity and dynamic elasticity as a function of time. This approach simplifies the methodology of modeling the knitting process in dynamic approach.

The aim of the publication is to demonstrate the usefulness of selected models for description of knitting processes on warp-knitting machines.

\section{Material and experimental research program}

Yarns that are most often processed on warp-knitting machines are synthetic silks -polyamide, polyester and viscose, while cotton and wool are rarely used.

Three types of yarns used on warp knitting machines with average needle gauge were selected for the tests:

- polyester silk with linear mass density 84 dtex/f 48 (breaking force $\mathrm{P}_{\mathrm{b}}=3.4 \mathrm{~N}$, breaking elongation $\varepsilon=22.4 \%$ ),

- polyester silk with linear mass density 110 dtex/f 144 (breaking force $P_{b}=4.1 \mathrm{~N}$ breaking elongation $\varepsilon=26 \%$ ), 
- polyester silk with linear mass density 334 dtex/f 64 , (breaking force $P_{b}=11.0 \mathrm{~N}$, breaking elongation $\varepsilon=20.5 \%$ ).

Thread stretching speeds adopted for the tests result from the assumed knitting speeds $-n=700$ courses $/ \mathrm{min}$.

Stretching speed was calculated using the formula:

$$
V_{r}=\frac{\Delta l}{T_{i}}, m / s
$$

where: $\mathrm{Ti}$ - rise time of the impulse of stretching force, $\Delta \mathrm{l}$ - maximum thread displacement for the warp-knitting machine.

Maximum threads displacement was determined (equal to $\Delta l=5.6 \mathrm{~mm}$ ) between the back-rest and the needle comb for one course-forming cycle on warp knitting machine $\mathrm{K} 2$ by $\mathrm{K}$. Mayer with needle gauge $\mathrm{E}=22^{\left[{ }^{[2]}\right.}$

In order to calculate the stretching speed, the following parameters were determined:

- time of forming one knitted course

$$
T=\frac{60}{n}, s
$$

where: $\mathrm{n}$ - knitting speed courses/ min.

- impulse rise time

$$
T_{i}=\frac{T}{K}, m s
$$

where: $\mathrm{K}$ - average number of tension changes.

To calculate the impulse rise time, five cycles of tension changes were adopted, for which $K=10$. It takes account of five parts of rising phase and five parts of falling phase of force in the threads during the formation of one course on warp knitting machines with needle gauge $E \in[20,28]$ at knitting speeds, in the range from 600 to 1200 courses/min.

On the basis of the adopted stretching speeds, rotational speed of the extending arm of the device was calculated using the formula:

$$
n_{p}=\frac{V}{2 \pi r} \cdot 60, \text { courses } / \mathrm{min}
$$

where, $r$ - length of extending arm which equals $r=210 \mathrm{~mm}$.
The results are shown in Table 1.

The differences between the calculated and preset speed $n_{p}$ result from limited possibility of setting rotational speed with accuracy to tenths of a revolution.

The following lengths of stretched yarn sections were adopted for the study:

$I=400,700,1000$ and $1300-$ which reflects the most common distances between the beam and the back-rest, as well as the back-rest and the guide needle bar. ${ }^{[3]}$

The tests were performed with initial tension in the range from $5 \mathrm{cN}$ to $10 \mathrm{cN}$.

\section{Research Methodology}

An original device constructed at the Department of Knitting Technology of Lodz University of Technology was used for testing the process of dynamic stretching of yarns. A diagram of the measuring unit is shown in Figure 1.

The measuring stand was built of a strain gauge (5) to which one end of the yarn was fixed. Initial tension $\mathrm{P}_{0}$ was set by shifting the sensor in the movable holder to the value of $10 \mathrm{cN}$, controlled on the indicator of tensometric amplifier. The other end of the yarn was stuck in the movable clamp (4). Between the movable clamp and the sensor, the yarn was arranged horizontally. The device was constructed with a flywheel (1), equipped with an arm (2) and an arbor (2'), causing displacement of the movable clamp by a fixed length, $\Delta l=24 \mathrm{~mm}$. After attaching the yarn, the device driving the flywheel was set in motion. The stretching speed was adjusted by turning the knob (7). Setting an appropriate rotational speed made the arbor end gain linear stretching speeds consistent with the values shown in Table 1. At the moment the arbor was in the upper position, it was released by pulling out a pin and the program recording information on the stretching process was launched simultaneously. During rotation, the released pin hit the buffer causing a displacement of the movable clamp and yarn stretch. The buffer was stopped by a permanent magnet. Different lengths of the stretched yarn sections were set by changing the position of the strain gauge. Changes in yarn tension caused changes of voltage in the measuring circuit, which, strengthened by the tensometric amplifier and after being processed by the analog-digital interface were

Table 1. Summary of calculated values $/ 1,2,3,4 /$

\begin{tabular}{|c|c|c|c|c|c|}
\hline $\begin{array}{c}\text { Knitting speed } \\
\mathbf{n}, \text { course/min. }\end{array}$ & $\begin{array}{c}\text { Formation time } \\
\text { of 1st knitted } \\
\text { course T, s }\end{array}$ & $\begin{array}{c}\text { Impulse rise } \\
\text { time } \mathbf{T}_{\mathbf{i}}, \mathbf{m s}\end{array}$ & $\begin{array}{c}\text { Stretching } \\
\mathbf{s p e e d} \mathbf{V}_{\mathbf{r}}, \mathbf{m} / \mathbf{s}\end{array}$ & $\begin{array}{c}\mathbf{n}_{\mathrm{p}} \text { rotational speed } \\
\text { of extending arm } \\
\text { (calculated), r/min. }\end{array}$ & $\begin{array}{c}\mathbf{n}_{\mathbf{p}} \text { rotational speed of } \\
\mathbf{e x t e n d i n g} \text { arm } \mathbf{p r e s e t} \\
\mathbf{d u r i n g} \text { the test), } \\
\mathbf{r} / \mathbf{m i n} .\end{array}$ \\
\hline 700 & 0.086 & 8.6 & 0.65 & 29.55 & 30 \\
1200 & 0.050 & 5.0 & 1.12 & 50.91 & 50 \\
1600 & 0.038 & 3.8 & 1.47 & 66.82 & 67 \\
\hline
\end{tabular}




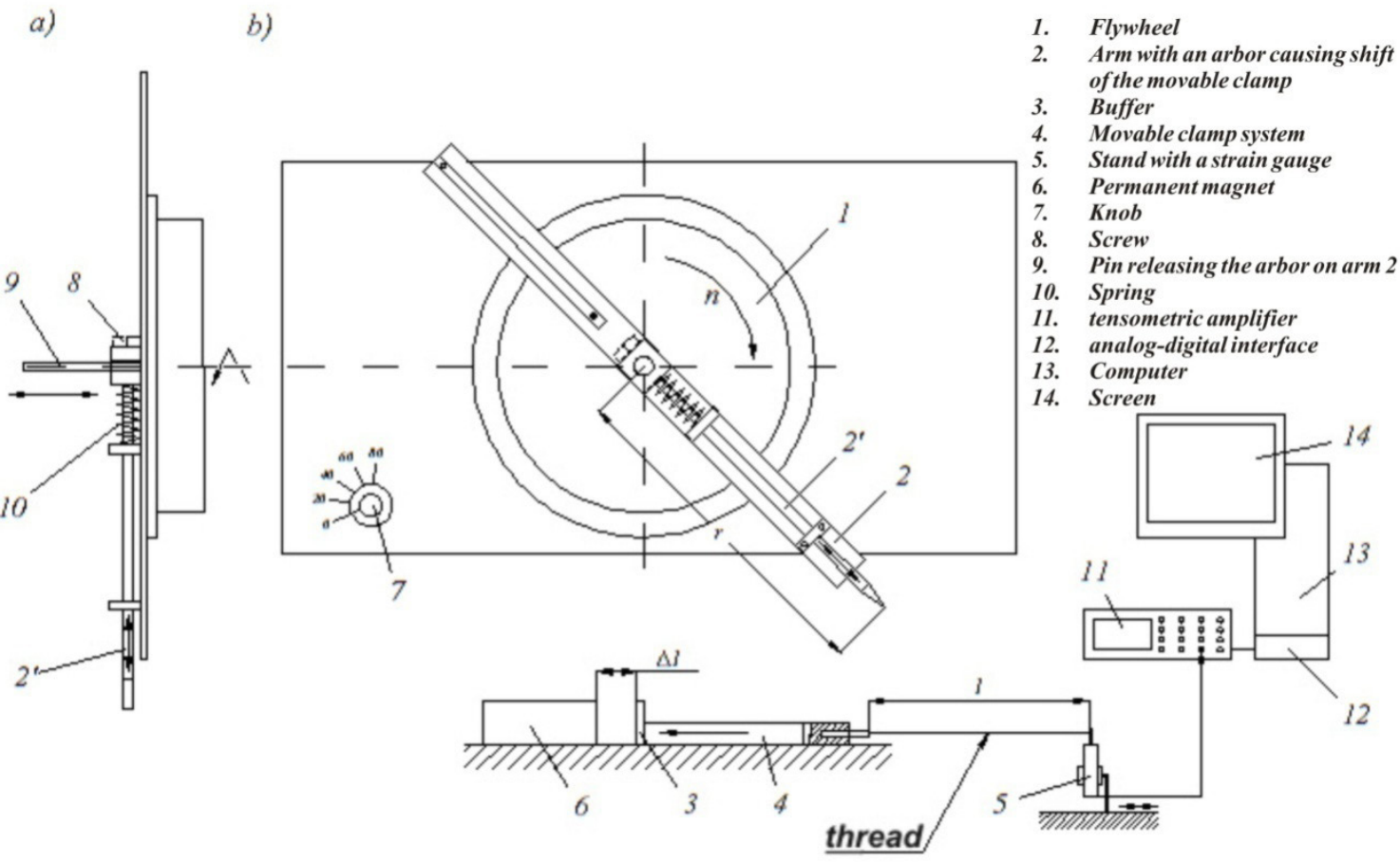

Figure 1. Diagram of measuring unit: a) side view, b) front view

graphically represented on the computer in the form of $U=f(t)$ charts.

During one measurement, 10,000 points of tension value were recorded in $649 \mathrm{~ms}$, which corresponds to the sampling time of $65 \mu$ s for one measurement channel.

From a series of several measurements performed for a given variant, the averaging characteristic of the forces in the thread as a function of time was digitally elaborated.

The tensions obtained during testing dynamic yarn stretch were recalculated from the values in $\mathrm{mV}$ into $\mathrm{cN}$, according to a linear relationship.

\section{Determining elasticity coefficient $E_{K}$ for kinematic loading}

In order to determine the elasticity coefficient $E_{K}$ for kinematic loading at thread stretching speed $\mathrm{d} \varepsilon / \mathrm{dt} \rightarrow 0$, the yarns used in the test were subjected to stretching by Instron tensile testing machine, type ZWICK 1120. ${ }^{[4]}$

The parameters of stretching process:

- stretching speed $\mathrm{V}=10 \mathrm{~mm} / \mathrm{min}$. - initially, the adopted stretching speed was $V=2 \mathrm{~mm} / \mathrm{min}$; ; however, because of very long stretching time, about 1 hour, the speed was increased to $10 \mathrm{~mm} / \mathrm{min}$.

- length of stretched yarn section: I $=500 \mathrm{~mm}$,

- initial tension $\mathrm{P}_{0}=3 \mathrm{cN}$.

As a result of the study, the dependence of force as a function of elongation was obtained. As a result, for each type of yarn, two values of elasticity coefficient $E_{k 1}$ and $E_{k 2}$ were obtained and so:

for yarn PE $84 \mathrm{dtex}-\overline{E_{K 1}}=2434.54 \mathrm{cN} \overline{E_{K 2}}=1680.43 \mathrm{cN}$ for yarn PE $110 \mathrm{dtex}-\overline{E_{K 1}}=4020.16 \mathrm{cN} \overline{E_{K 2}}=2327.38 \mathrm{cN}$ for yarn PE 334 dtex $-\overline{E_{K 1}}=8358.17 \mathrm{cN} \overline{E_{K 2}}=5985.05 \mathrm{cN}$

\section{Results and analysis}

The next research stage was the analysis of rheological models of threads, ${ }^{[5-11]}$ stretching in terms of comparing the real characteristics with the model ones.

The analysis was performed on the basis on four rheological models:

1. two-parameter Maxwell model,

2. two-parameter Kelvin-Voigt model,

3. standard three-parameter Zener model 1,

4. standard three-parameter model 2.

Elasticity coefficient Ed was determined from the obtained measurement results of dynamic loads of threads. This coefficient is not present in Kelvin-Voigt model, and for the other models, it was determined from the following relationships:

- for Maxwell model and Standard model 2

$$
\mathrm{Ed}_{\mathrm{m}}, \mathrm{Ed}_{\mathrm{s}}=\frac{P_{\max }}{\varepsilon}, \mathrm{cN} \text {, }
$$




$$
\begin{aligned}
& \mathrm{Ed}_{\mathrm{Z} 1}=\frac{P_{\max }}{\varepsilon}-\mathrm{E}_{\mathrm{K} 1}, \mathrm{cN}, \\
& \mathrm{Ed}_{\mathrm{Z} 2}=\frac{P_{\max }}{\varepsilon}-\mathrm{E}_{\mathrm{K} 2}, \mathrm{cN},
\end{aligned}
$$

where: $E_{K}$ - elasticity coefficient for kinematic loading at thread stretching speed $\frac{d \varepsilon}{d} \rightarrow 0$

Viscosity coefficient $\eta$ was determined using the formulas:

$$
\begin{aligned}
& \mathrm{\eta}\left(\mathrm{E}_{\mathrm{K} 1}\right)=\frac{P_{\text {max }}}{\omega}-\frac{E_{K 1} \cdot \varepsilon}{\omega}, \mathrm{cNs}, \\
& \mathrm{\eta}\left(\mathrm{E}_{\mathrm{K} 2}\right)=\frac{P_{\text {max }}}{\omega}-\frac{E_{K 2} \cdot \varepsilon}{\omega}, \mathrm{cNs},
\end{aligned}
$$

Conducting comparative analysis of real and model characteristics required by creating a spreadsheet in Microsoft Excel.

All the theoretical and empirical data were interpreted graphically, showing curves of the threads stretching models and real force as a function of time.

Comparative analysis was based on a proper selection of the coefficients for individual rheological models, so that their curves reflect the shape of the real curve as accurately as possible.

Each variant was examined according to the following criteria:

a. the analysis included the input parameters determined for $E_{K 2}$, while the viscosity coefficient was not changed,

b. input parameters were the same as in case a, and viscosity coefficient was specially selected,

c. the analysis included input parameters determined for $\mathrm{E}_{\mathrm{K} 1}$, and viscosity coefficient was specially selected.

\subsection{Comparative analysis of empirical and mode characteristics}

Comparisons of empirical and model characteristics were performed for three stretching speeds: $V_{1}=0.65 \mathrm{~m} / \mathrm{s}$, $\mathrm{V}_{2}=1.12 \mathrm{~m} / \mathrm{s}, \mathrm{V}_{3}=1.47 \mathrm{~m} / \mathrm{s}$, depending on the length of the stretched thread section.

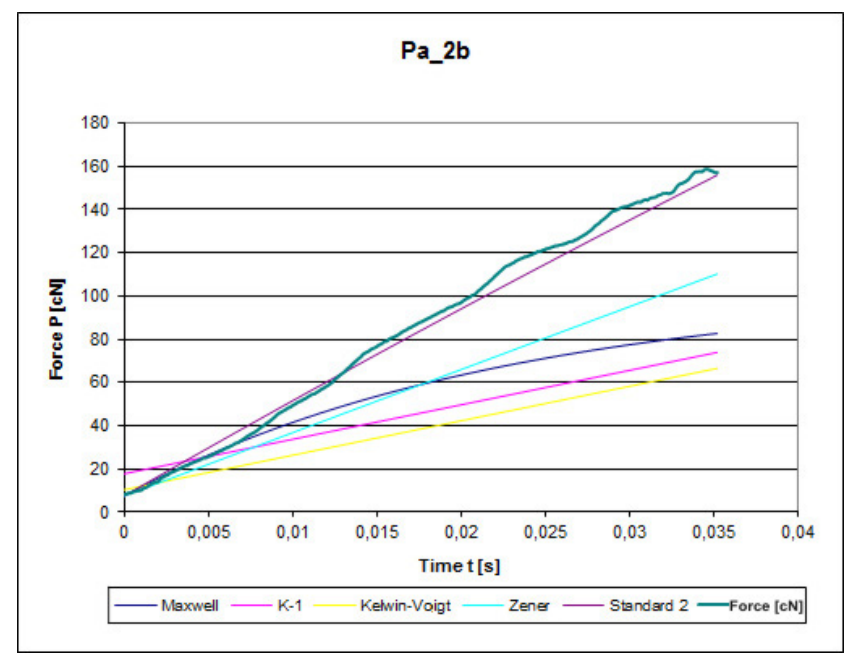

Figure 2. Model characteristics and real values for variant $\mathrm{Pa}$ _2b (PE 110 dtex, I = $1000 \mathrm{~mm}, \mathrm{~V}=0,65 \mathrm{~m} / \mathrm{s}, \mathrm{E}_{\mathrm{K} 2}=2327,38 \mathrm{cN}$ )
From the conducted comparative analysis of theoretical models and empirical characteristics of force changes as a function of time, for threads stretched with three stretching speeds, it can be concluded that:

- in most cases, the curve of Standard model 2 best reflects the shape of the empirical curve (Fig. 2 and Fig. 3),

- the curve of Zener model is similar to the real characteristic for longer sections of stretched yarns $\mathrm{I}=1000 \mathrm{~mm}$ and $\mathrm{I}=1300 \mathrm{~mm}$, and in one case, for $\mathrm{I}=700 \mathrm{~mm}$ and $\mathrm{V}=1.47 \mathrm{~m} / \mathrm{s}$,

- the characteristic of Kelvin-Voigt model shows convergence with empirical characteristic only for I $=1300 \mathrm{~mm}$; while for $\mathrm{I}=400 \mathrm{~mm}$, only the curve of Zener model is similar to the real characteristic,

- the curve of Maxwell model diverges from the empirical curve in all variants,

- model characteristics get closer to the empirical curve when stretching speed increases,

- in case of the shortest stretched section I $=400 \mathrm{~mm}$, the real curve is most non-uniform and there is large discrepancy between the curve of Standard model 2 and the real characteristic,

- the influence of yarn thickness on the character of real curve and its convergence with model characteristics is negligible.

\subsection{Comparative analysis of empirical and model characteristics for shortened time of yarn stretching}

The second stage of the analysis was performed for 22 selected variants:

The shortened stretching time was determined by substituting for $\Delta \mathrm{l}$, the value of maximum thread displacement on warpknitting machine $\mathrm{K} 2(\Delta \mathrm{l}=5.6 \mathrm{~mm})$.

The analysis concerned variants with variable lengths of the stretched sections, variable stretching speed and thread thickness. Each variant was analyzed for two $E_{K}$ values $\left(\mathrm{E}_{\mathrm{K} 1}\right.$ and $\left.\mathrm{E}_{\mathrm{K} 2}\right)$.

In the analyzed variants, the introduced characteristic was 'Standard 2 + formulas', which illustrates the equivalence

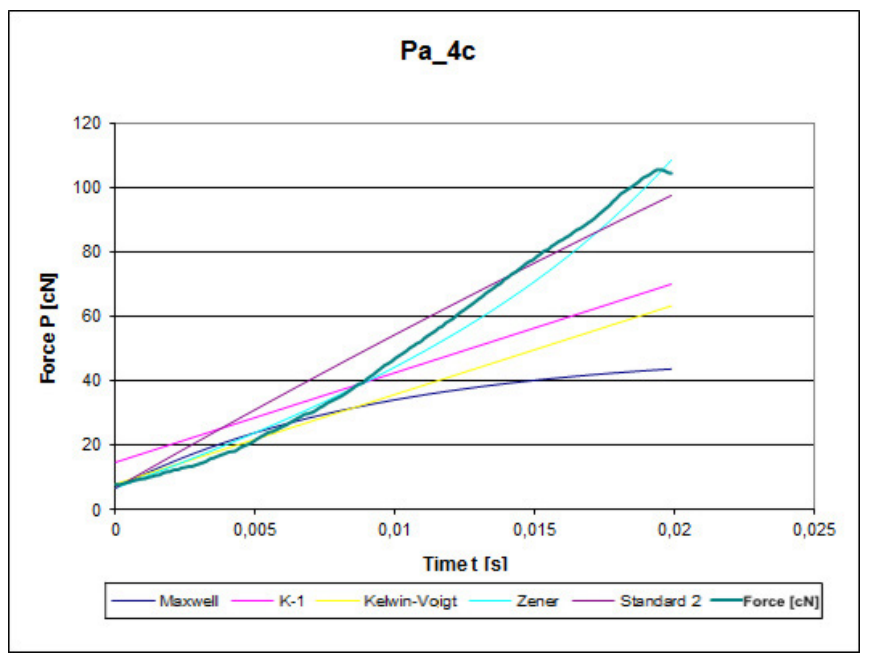

Figure 3. Model characteristics and real values for variant $\mathrm{Pa} \_4 \mathrm{C}$ (PE 84 dtex, I = $1000 \mathrm{~mm}, \mathrm{~V}=1,12 \mathrm{~m} / \mathrm{s}, \mathrm{E}_{\mathrm{K} 1}=2434,54 \mathrm{cN}$ ) 
of Zener and Standard 2 models. The characteristics of these models are equivalent, if the coefficients of Standard model 2 take the form:

$$
\begin{aligned}
& E d_{S t}=\frac{E_{2} \cdot E_{K}}{E_{2}-E_{K}} \\
& \eta=\eta_{\text {Zener }} \cdot \frac{E_{1}+E_{2}}{E_{1 Z}}
\end{aligned}
$$

It can be concluded that among the examined variants in eighteen cases, the Zener model and equivalent model 'Standard $2+$ formulas' best describe the stretching process (Fig. 4 and Fig. 5).

In addition, it should be emphasized that:

- while the stretching speed increases, the compatibility of theoretical characteristics and real curve also rises,

- better convergence of model characteristics and experimental curve can be observed for shorter sections of the stretched yarn $\mathrm{I}=400 \mathrm{~mm}$, than for $\mathrm{I}=1000 \mathrm{~mm}$.

Although model characteristics do not always perfectly reflect the nature of the experimental curve, their convergence with empirical characteristic is much larger than for elongation $\Delta \mathrm{l}=24 \mathrm{~mm}$.

\section{Mathematical equivalence of Kelvin-Voigt and Zener models}

Two cases of equivalence of Kelvin-Voigt and Zener models were examined for initial conditions of thread stretching $\mathrm{P}_{0}=0$ (no initial tension in the thread undergoing stretching) and for $\mathrm{P}_{0}>0$.

1. Initial conditions of thread stretching when $P_{0}=0$.

In Kelvin-Voigt model, in order to receive an equivalent form

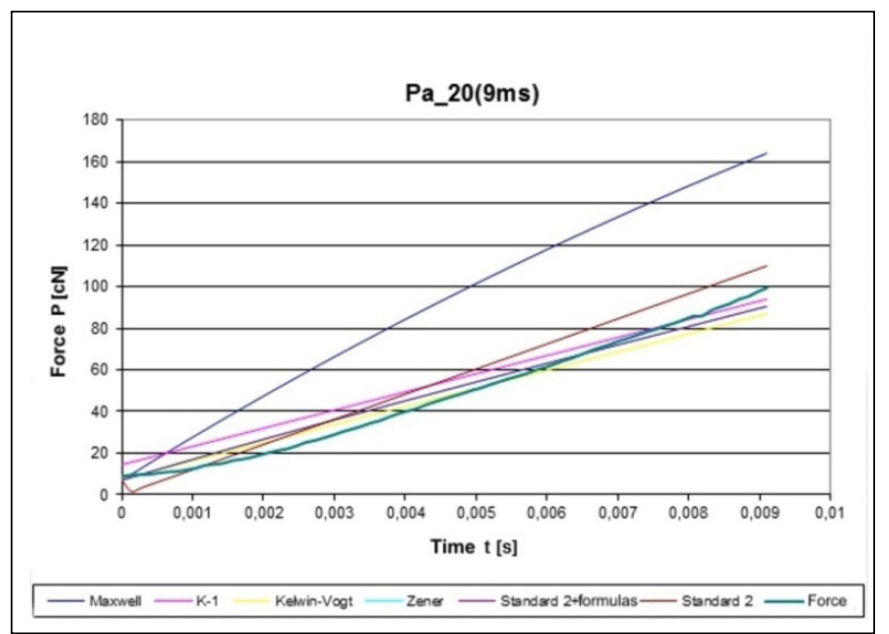

Figure 4. Model characteristics and real values for variant $\mathrm{Pa} 20 \mathrm{~b}$ (PE $110 \mathrm{dtex}, \mathrm{I}=400 \mathrm{~mm}, \mathrm{~V}=0,65 \mathrm{~m} / \mathrm{s}, \mathrm{E}_{\mathrm{K} 2}=2327,38 \mathrm{cN}$ ) of Zener model, the following equation of state is formulated:

$$
P(t)=E_{K} \cdot \omega \cdot t+k_{1} \cdot \omega
$$

where:

$$
k_{1}=\eta_{K}=\eta_{1(\text { Zener })} \cdot\left(1-\exp \left(\frac{-E_{1 Z} \cdot t}{\eta_{1}}\right)\right)
$$

2. Initial conditions of thread stretching when $\mathrm{P}_{0}>0$.

In Kelvin-Voigt model, in order to obtain an equivalent form of Zener model, the following equation of state is formulated:

where:

$$
P(t)=P_{0} \cdot k_{2}+E_{K} \cdot \omega \cdot t+k_{1} \cdot \omega
$$

$$
\begin{aligned}
& k_{1}=\eta_{1 \text { (Zener) }}\left(1-\exp \left(\frac{-E_{1 Z} \cdot t}{\eta_{1}}\right)\right) \\
& k_{2}=\exp \left(\frac{-E_{1 Z} \cdot t}{\eta_{1}}\right)
\end{aligned}
$$

In the considered variants of equivalence of Kelvin-Voigt and Zener models, the curve of Standard model 2 best reflects the real curve, just like in case of comparative analysis of empirical and model characteristics presented in section 5.1.

The curve of Zener model and overlapping characteristic of Kelvin 2, for the condition: $P_{0}=0$ and $K-3$ for $P_{0}>0$, are convergent with the experimental curve. The convergence of Zener and Kelvin 2 curve with the real characteristic is obtained after changing (increasing) the value of Ed coefficient determined by dynamic thread loads.

For two randomly selected variants, the results of equivalent systems of Zener and Kelvin-Voigt models are presented (Fig. 6a and 6b). In the graphs, the model curves overlap. Thus, while using the system of mathematical equivalence mentioned above, it can be concluded that these models accurately describe the dynamic stretching of the analyzed polyester threads in relation to the conditions of knitting process on warp-knitting machines.

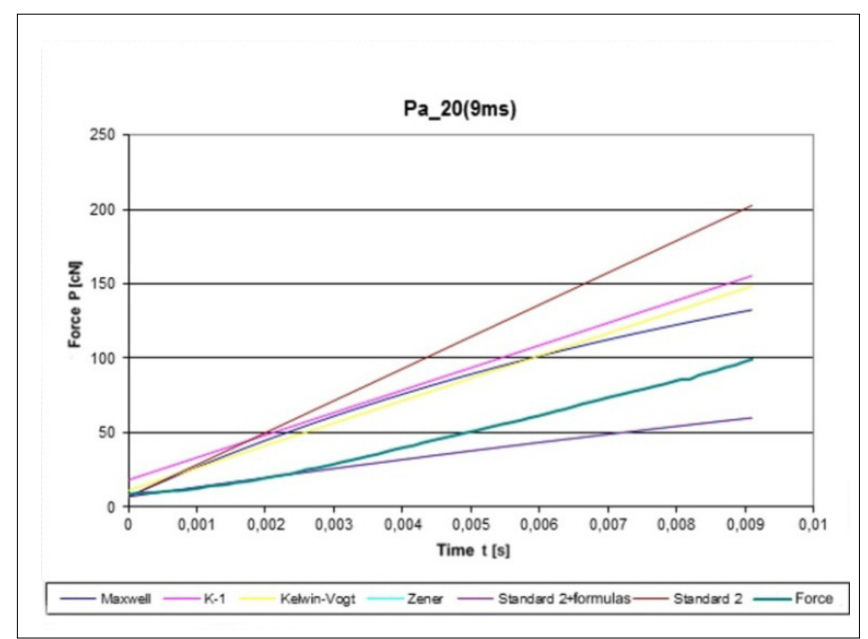

Figure 5. Model characteristics and real values for variant $\mathrm{Pa}$ 20c (PE 110 dtex, $\mathrm{I}=400 \mathrm{~mm}, \mathrm{~V}=0,65 \mathrm{~m} / \mathrm{s}, \mathrm{E}_{\mathrm{K} 1}=4020,16 \mathrm{cN}$ ) 

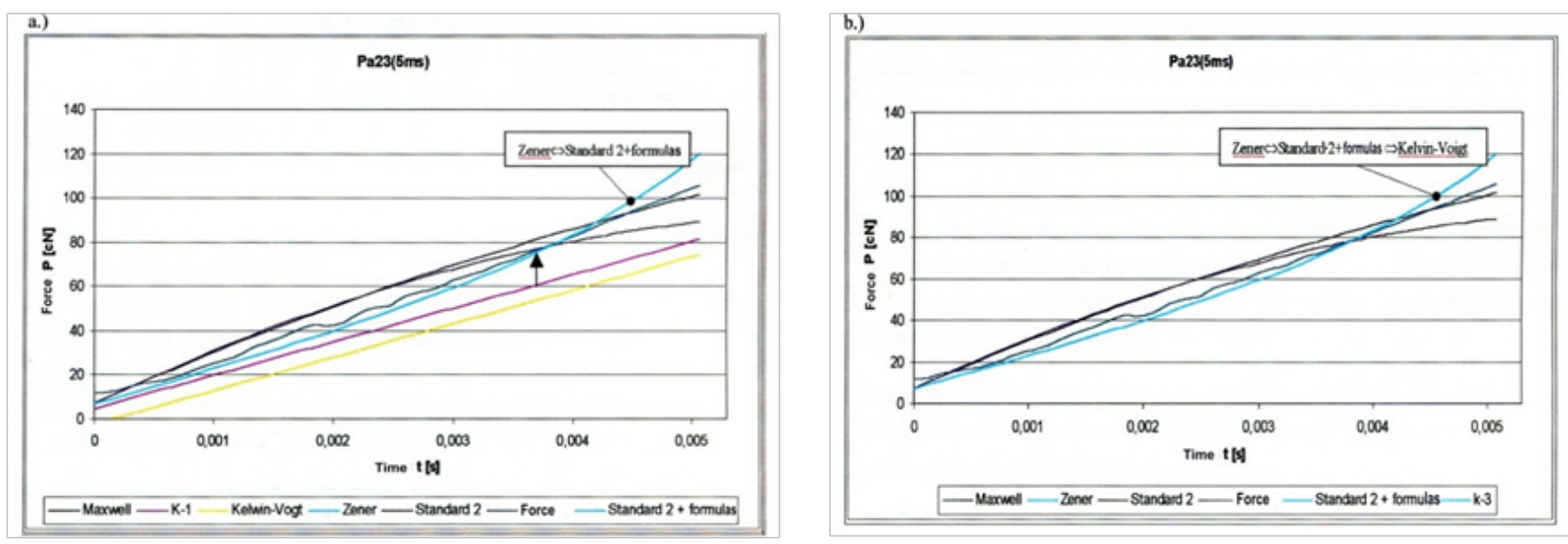

Figure 6. Model characteristics and real values for variant Pa_23 (PE $110 \mathrm{dtex}, \mathrm{I}=400 \mathrm{~mm}, \mathrm{~V}=1,12 \mathrm{~m} / \mathrm{s}$ ) a. Equivalence of Zener Standard 2 models

b. Equivalence of Zener Standard 2and Kelvin-Voigt models

\section{Conclusions}

1. As a result of a comparative analysis of rheological models and real curves of the process of stretching polyester threads with linear densities 84,110 and 334 dtex for the adopted deformation rates $\varepsilon(t) \in[0.53,4.00] 1 / \mathrm{s}$, large convergence was observed between Standard model 2 and empirical values. For some of the analyzed variants, Zener model curves correspond to the real characteristics. For three considered stretching speeds $(\mathrm{V}=0.65 ; 1.12$; $1.47 \mathrm{~m} / \mathrm{s}$ ), the curves of rheological models most accurately reflect the empirical curves for $\mathrm{V}=1.47 \mathrm{~m} / \mathrm{s}$. For the analyzed thicknesses of the stretched yarn, no impact of linear density was observed on the convergence between model and real characteristics.

2. Regarding the time period $t \in[0,9] \mathrm{ms}$, corresponding to the time of stretching threads on warp knitting machines with average needle gauge ( $E=20$, warp knitting machine $K 2$ ), real curves are most accurately described by Zener model and equivalent Standard model 2, if the coefficients in this model take the following form:

$$
E d_{S t}=\frac{E_{2} \cdot E_{K}}{E_{2}-E_{K}} \quad \eta=\eta_{\text {Zener }} \cdot \frac{E_{1}+E_{2}}{E_{1 Z}}
$$

Large divergence of model and empirical characteristics was observed for shorter sections of stretched yarns.

In a significant group of analyzed variants (15 out of 22), the characteristics of Zener model are convergent with those of Kelvin-Voigt model.

3. For the adopted systems of mathematical equivalence of Standard model 2 and Zener model, for independent constant of $\mathrm{Ed}_{\mathrm{St}}$ and $\eta$ models specified in point 2 and KelvinVoigt and Zener models for parameter:

$$
\eta_{K}=\eta_{1 \text { (zener) }} \cdot\left(1-\exp \left(\frac{-E_{1 Z} \cdot t}{\eta_{1}}\right)\right)
$$

The curves of Standard model 2, Zener and Kelvin-Voigt models overlap and for the determined elasticity, the coefficients Ed and $E_{K}$ and the selected viscosity coefficient $\eta_{\text {zener }}$ for Zener model, the models accurately describe the nature of the stretching process.

\section{References}

[1] Prążyńska A., Mikołajczyk Z. Identification of the process of dynamic stretching of threads in warp knitting technology. Part I: Theoretical analysis of the susceptibility of rheological models of the process of stretching textile threads. 2017, DOI: 10.1515/aut-2016-0039.

[2] Kopias K. Structure and technology of warp - knitted fabrics, copyright by Lodz University of Technology 2010, $188 p$.

[3] Mikołajczyk Z. Identification of knitting processes of anisotropic warp-knitted structures produced on warpknitting machines. (in Polish) Scientific Notebook nr 1047, copyright by Lodz University of Technology 2009, 234 p.

[4] PN-EN ISO 2062:1997 Textiles. Yarns from packages. Determination of single-end breaking force and elongation at break.

[5] DerskiW.,ZiembaS.Analysisofrheologicalmodels. (inPolish) Polish Scientific Publishers PWN, Warsaw 1968, Issue I.

[6] W. Kobza, J. Gluza, Mechanics and technical rheology, Script for universities (in Polish), Technical University of Lodz, Lodz, 1991.

[7] G. Urbańczyk, Physics of fibre (in Polish) Technical University of Lodz, Lodz, 2002.

[8] Barnes H.A., Hutton J.F., Walters K. An introduction to rheology. Elsevier science Publishers B. V., ISBN 0-44487140-3, 1989, $201 p$.

[9] Moczo P., Kristek J. \& Franek P. Lecture Notes on Rheological Models. DAPEM FMPI CU, Bratislava 2006.

[10] Mainardi F., Spada G. Creep, Relaxation and Viscosity Properties for Basic Fractional Models in Rheology, The European Physical Journal, Special Topics, Vol. 193 (2011) 133-160.

[11] Marques S. P. C and Creus G. J., Computational Viscoelasticity, Springer Briefs in Computational Mechanics, DOI: 10.1007/978-3-642-25311-9_2, 2012, $124 p$. 